\title{
ARM-embedded Biofeedback Training System
}

\author{
Yong-Fang Ling $\mathrm{Hu}^{1, \text { a }}$, Heng Shu ${ }^{2, b}$ \\ ${ }^{1}$ Guizhou Colloge of Finance and Economics ,Guiyang ,China \\ ${ }^{2}$ Guizhou Normal University, Guiyang, China \\ ahyongfang@aliyun.com, ${ }^{\mathrm{b}}$ 656163119@qq.com
}

Keywords: ARM; psychological adjusting ;biofeedback; relaxation-training

Abstract. This paper designed a kind of psychological adjusting feedback control system based on ARM. This system is composed of S3C2410 micro controller and its peripheral circuit. Using the embedded system platform, and discusses when sampling object anxiety and other psychological state automatically to the psychological adjustment, and through the remote monitoring and control system can make psychological experts always understand sampling the state of an object for remote voice and text communication makes it possible to be targeted.

\section{Introduction}

With the deepening of the human biology research, biological cycles (physical, emotional and intellectual) are gradually revealed. But because of the biological features, each individual organism cycle and frequency does not remain at the same time with the same period. Each independent individual organisms have different cycle and frequency. So how to make an organism in a particular time with the best biological characteristics, adjustment of both biological individual biological cycle and frequency, the people's will have a certain role in promoting the regulation of physiological function.

Produced the biofeedback therapy in the $1930 \mathrm{~s}$, it is the application of modern science and technology will usually people don't realize the biological signal [1], such as electrical, electrical, galvanic skin, heart rate, blood pressure, etc., into a people can be aware of the signals, such as hearing, vision information, training according to these signals to learn in a certain range consciously control the activities of the internal organs, correct the deviation from the normal activities of the internal organs treatment and training methods $[2,4]$. Biofeedback mainly have three: the principle of the system theory, operant conditioning, information theory and cybernetics, which is mainly on the logic for the interpretation of the biofeedback [3]. Its development can be roughly divided into three stages: the first stage is the $1960 \mathrm{~s}$, researchers realized on single or multiple motion unit inactivation and activation of control; The second stage is the $60 \mathrm{~s}$ and ninety s last century, found the operant conditioning theory of autonomic nervous system, laying the theoretical basis of biological feedback treatment of liver disease, to establish the professional organization of biofeedback; The third stage is the $1990 \mathrm{~s}$ to the present, because the brain electrical experts found that the number of different frequencies of brain waves, and people's consciousness, emotional highly correlated, the nerve biofeedback, researchers also began in neurophysiology explore the specific effect of biological feedback mechanism on the basis of $[3,4]$. Now biofeedback is widely used in clinical, its validity is confirmed by many. But its training efficiency to a great extent, relies on the professional level of biofeedback trainers and patients suggested capacity [4].

Designed in this paper based on ARM embedded biofeedback training system is based on the principle of biofeedback, USES the ARM microprocessor, embedded system as a whole operating system, the control of the individual information center not only meet the real-time control of the external device, also meet the powerful network function, achieve the function of multiple physiological parameters, simple and portable, powerful intelligent biofeedback system. By regulating the biological cycle, make its can play the best in peak performance. And people pay more and more attention to the healthy and stable physiological function, in order to realize the 
psychological adjustment, improvethe nervous and anxious state of mind, to better face the pressure for work and life in modern society.

\section{Feedback training system architecture design}

Signal adjustive module. The unit mainly complete the front end of the data acquisition, biological information can be converted to digital signals. Because of the human body physiological signal belongs to the weak non-stationary signals, the frequency and amplitude is low, vulnerable to interference. Need on corresponding processing to the collected signals, in order to better restrain all kinds of interference, undistorted physiological signal amplification.

This module is mainly responsible for signal acquisition, filtering and amplification, and converts analog signals into the ARM micro controller can be directly introduced into digital signals, the system composition is shown in figure 1: it is mainly composed of sensors, amplifying circuit, filter of trap circuit, A/D converter.

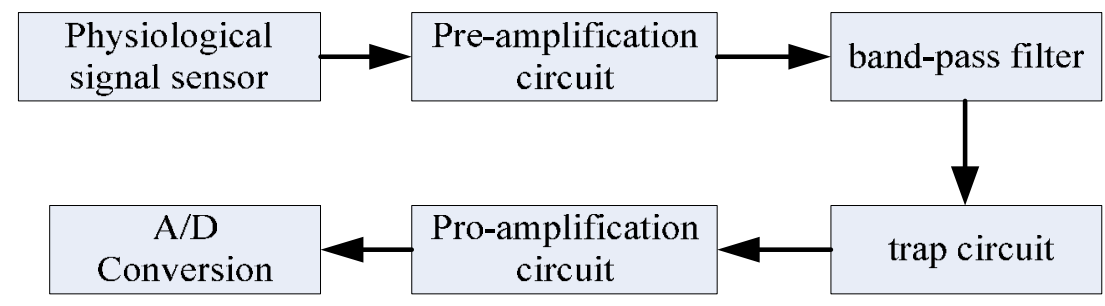

Fig. 1 Signal collection and processing circuit

Front and after the implantation of large circuit adopts AD company instrument amplifier AD620, bandpass (composed) high pass and low pass filter and trap circuit composed, low-noise NE5532 dual operational amplifier parameters acquisition module selects 24 sampling resolution of 8 channel fast A/D chip ADS1216 [5,6].

Transfer ARM microcontroller and display unit. ARM microcontroller and display unit as shown in figure 2, Hardware choose SAMSUNG company as PDA, Internet equipment, handheld terminals, such as development of S3C2410[7] processor, frequency up to $266 \mathrm{MHZ}$, internal provide abundant peripheral interface. Through transplantation Wince operating system, constitute a complete embedded systems.

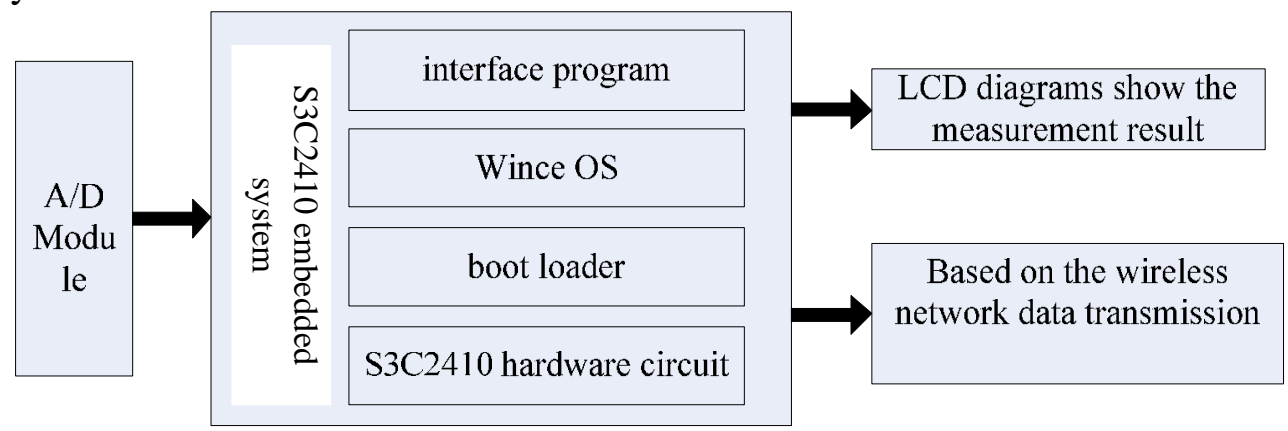

Fig.2 ARM Processor

Embedded terminal analog signals by the AD conversion, the GUI using EVC, development and design based on the EVC application interface, the multi-channel acquisition results in the form of data/time chart real-time display on the LCD, real-time analysis and comparison for users.

Software architecture. Due to the biological training feedback way according to the comprehensive evaluation of the acquisition object, thus guiding experts need to the analysis of the collected data to carry on the effective guidance, to complete the feedback. Based on this kind of works, this system uses C/S structure, namely the client to complete the data collection and analysis of statistical and basic feedback (video, audio, game), the server to complete the guidance of the object to be sampled voice, text, and to all the client's management. Figure 3 is the physical composition of this system. 


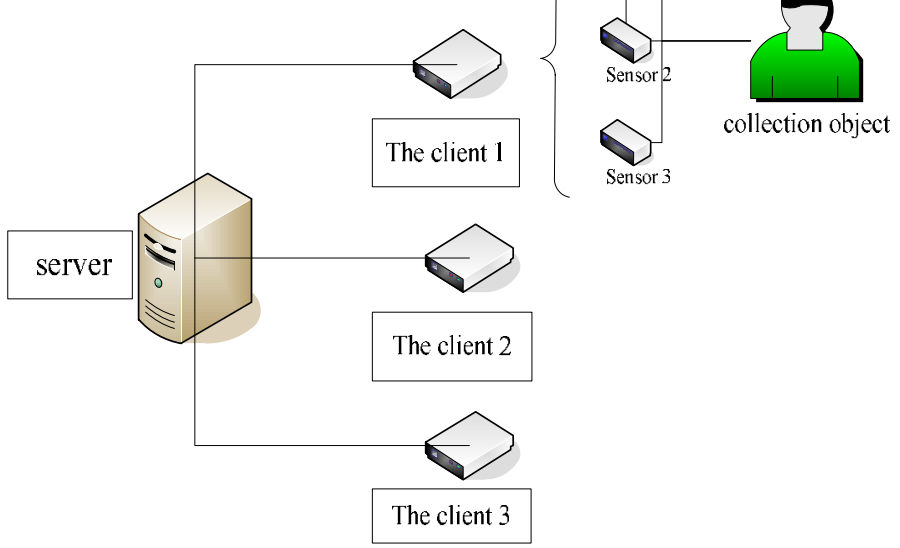

Fig 3. The physical structure mode

Biological training feedback system, which is based on the embedded system development, mainly based on multiple data fusion technology in the form of a message flow main key technologies are multithreaded, sockets, critical area, data lock, data filtering, data integration, data classification, data analysis, double buffer mechanism, etc.

Because the system uses the dynamic tracing analysis approach to feedback, therefore have greater difficulty in data synchronization and collaboration, communication and synchronization between threads.

Server modules:

The module to complete data communication interaction and other related information, and multiple clients for comprehensive management, etc. The logic relations as shown in figure 4.

a) UDP connection: Because the way of speech signal using TCP packets, will lead to huge delay of data, so the UDP to complete with the client connection, to complete the voice packets sent.

b) UDP send: Complete with speech signal sent via Udp client data interaction.

c) Speech signal analysis: Through the analysis of the speech signal receiving, speech signal, and wavelet transform is adopted to improve the filtering parting, in order to achieve ideal speech signal.

d) speech signal compression : As a result of the speech signal data volume is relatively large, so th e data compression will greatly reduce the network congestion probability, the system adopts the most popular g9276 compression algorithm, data compression and decompression.

e) TCP monitor: To monitor the server via TCP can know when the client access to the server, and along with all the client information.

f) TCP connection: Server via a TCP connection can communicate with each client by independent, without multiple client access, multiple clients data occurred.

g) Voice signal sampling: Through multi-thread mechanism, to activate the microphone, sampling microphone data, and data lock, the data into the critical region, finally sent via Udp, sends the data to the specified client.

h) Date transmit-receive: Through a TCP connection, complete the data, the link between control a nd sending data transceiver completion status, and chat, physiological signal data transceiver.

i) Voice prompt: Through multi-thread mechanism, activate the sound, through the collected data, and data lock, the data into the critical region, the last broadcast speech. 


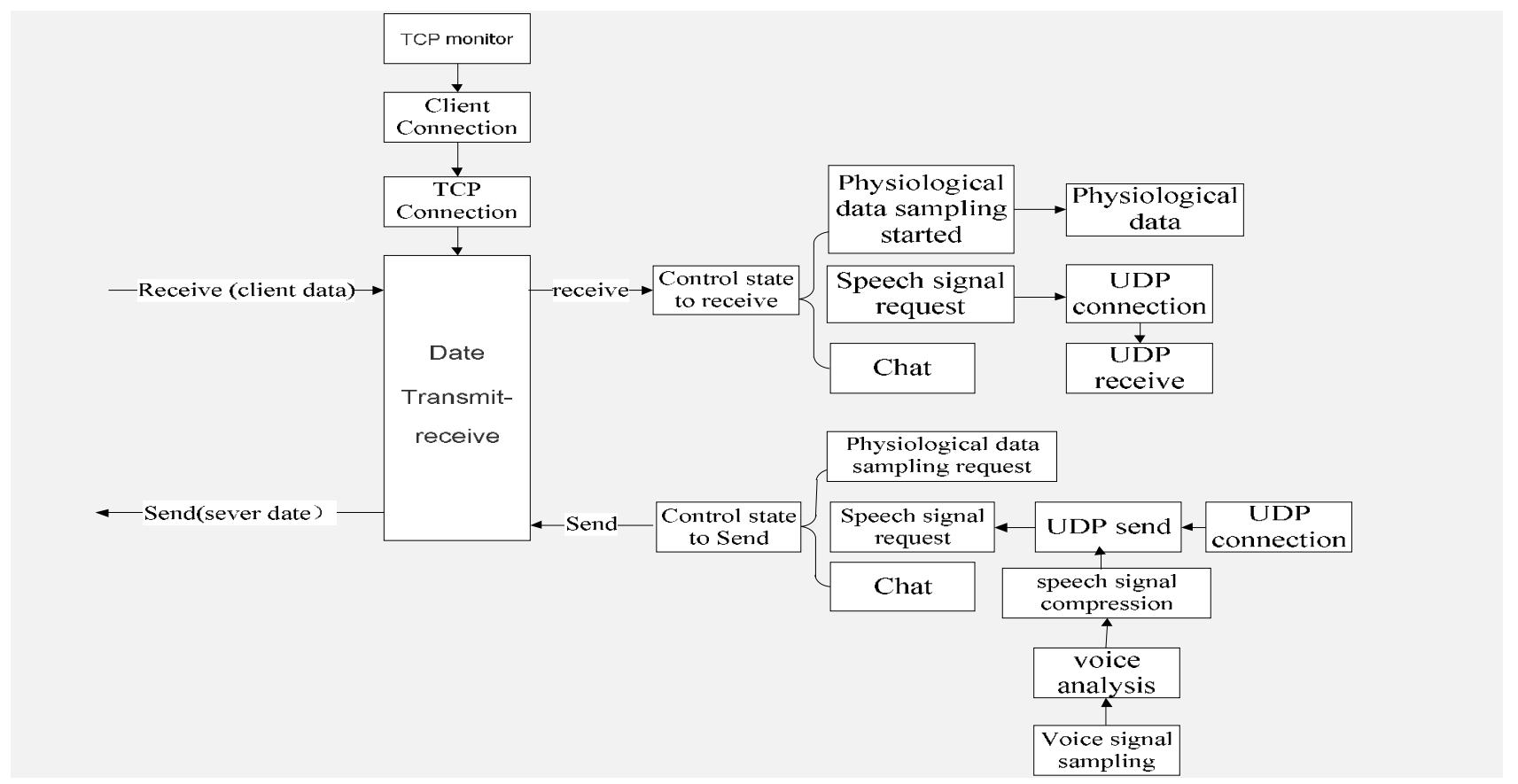

The client modules:

Fig 4. The server module logic relations

The module to complete the determination of biological signal acquisition and biological signal video audio feedback system startup management etc. The logic relations as shown in figure 5 .

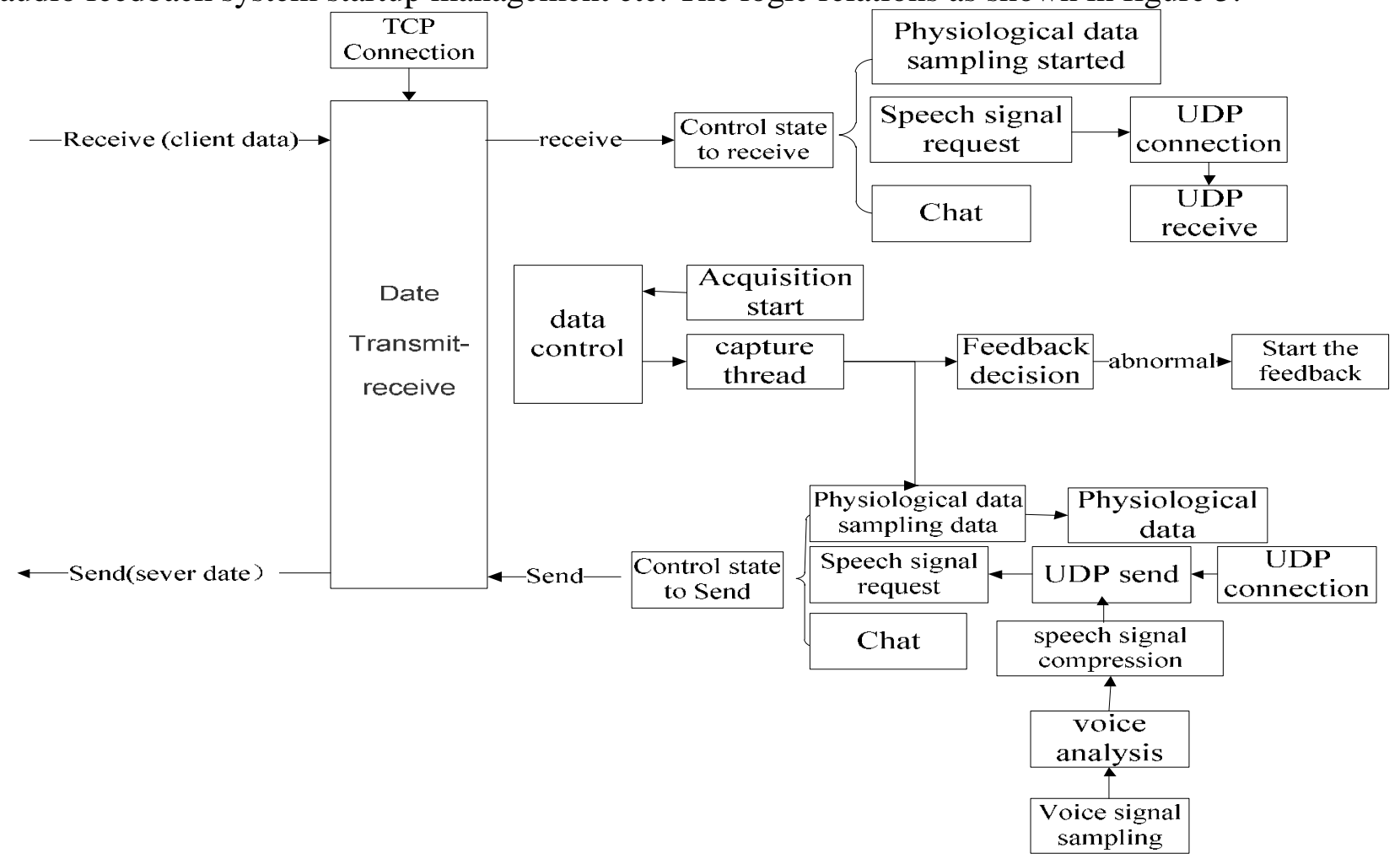

Fig5. The client module logic relationship

\section{Biofeedback training remote control system}

Biofeedback relax the intelligent system. Relax in the system of intelligent design can be instant for athletes, mental illness and all social classes provide the subconscious and consciousness induced visual music therapy module of mental relaxation, and can realize the human body physiological parameters of synchronous display, assist the individual psychological feedback effectively, increase the effect of psychological feedback[8]. 
Human body physiological parameters more all-round monitoring. Human body physiological parameters more comprehensive monitoring is the system key, through the real-time detection of the body's physiological parameters, suggested by real-time feedback and relax to strong individual psychological intervention, reduce the tension of the individual to achieve the purpose of relaxing.

To set up the remote control system. Through the embedded system, will at the bottom of the measurement and control device to connect to the Internet, realize remote measurement and control net-work.

\section{Experimental result}

A total of 60 students volunteers took part in the research, random divided into visual auditory feedback, comprehensive feedback group (visual + sound) and no feedback 3 groups, under the condition of their $8 \sim 12$ times feedback relaxation training. Experimental results found that: the way of feedback system of volunteers in physiological signal returned to normal after a certain period of time, without using feedback although physiological signals are also tend to be normal but the process and the way of time is the feedback of medicine is slow. Therefore using the feedback of this system can be a very good the body's physiological and psychological adjustment. But we also found from the experimental results of visual, hearing, and comprehensive feedback group the individual difference is bigger, is both an individual's cognition to different ways of feedback.Taken together, to individual differences in cognitive degree of comprehensive feedback less affected, greatly influenced by the audio and video mode.

Although this system can be a very good solution to biological psychological changes of anxiety,stress, such as, but because this system uses a physiological signal value preset.And as a result of individual differences determine abnormalities. Our next step will be in anomaly caused by individual differences.

\section{References}

[1] Yan-xia wu, lan-shuang wang. Electromyography feedback relaxation training effect on the treatment of students insomnia [J], journal of hebei normal university (natural science edition), 2008 (05).

[2] Gehrke; Dong Rui; Zhang wei different feedback forms of biofeedback and music relaxation training on physiological and psychological indexes $[\mathrm{J}]$ the influence of sports science, 2010, (4)

[3] YanHengChan Ding Xueqin, Chen Yongjia. Biofeedback technology application in the athletes' training process $[\mathrm{J}]$ sports and science, 2000, (03)

[4] Song Yulei; Lin Zheng; The; Mei-feng wang; Hong-jie zhang, biofeedback therapy joint comprehensive nursing intervention of clinical symptoms and quality of life in patients with functional constipation $[\mathrm{J}]$, the influence of the Chinese nursing journal, 2012, (6)

[5] Lu Changlong. Arm9-based bending machine controller of research and development [D]. Master degree theses of master of China excellent full text database, 2009 (05)

[6] Guo Ying; Chun-sheng ye; Intelligent temperature control system based on ARM [J], automation technology and applications, 2013, (02)

[7] Zhigang wang, wang north, LuoLeiFei. An embedded software component of the NFA quantitative measurement model [J]. Computer engineering, 2006, (13)

[8] Jia-qing wang, wu and achievements. G. 723.1 speech codec DSP real-time implementation [J] China data communications, 2005. (01). 